\title{
The use of metacognitive writing-to-learn prompts in an engineering statics class to improve student understanding and performance
}

\section{Dr. Saryn R. Goldberg, Hofstra University}

Dr. Saryn R. Goldberg is an Associate Professor of Mechanical Engineering in Hofstra University's School of Engineering and Applied Sciences. Dr. Goldberg received her Sc.B. in Engineering with a focus on materials science from Brown University, her M.S. degree in Biomedical Engineering with a focus on biomaterials from Northwestern University, and her Ph.D. in Mechanical Engineering with a focus on biomechanics from Stanford University. At Hofstra she teaches courses in mechanical engineering, materials science and biomechanics. In addition to her research in engineering education, Dr. Goldberg studies the biomechanics of human movement, focusing on gait rehabilitation. She is a member of the Society of Women Engineers and the American Society of Biomechanics.

\section{Dr. Jennifer Andrea Rich, Hofstra University}

Jennifer A. Rich received her doctorate in English and American literature at the Graduate Center/CUNY in October 2002. Her dissertation, Shakespeare's Economic Unconscious: Representations of Emergent Capitalism in Shakespeare's Drama, considers how the early modern emerging market-based economy is represented in Shakespeare's drama.

Rich has published widely in film, cultural studies, Shakespeare, rhetoric, critical theory and writing studies. She is Associate Professor of Writing Studies and Composition at Hofstra University.

\section{Dr. Amy Masnick, Hofstra University}

Dr. Amy Masnick is an Associate Professor of Psychology at Hofstra University. Dr. Masnick received both her B.S. and Ph.D. in Human Development at Cornell University. At Hofstra she teaches courses in introductory psychology, research methods, cognitive psychology, and child development. Dr. Masnick is interested in conceptual development, reasoning about science and number in children and adults, and in science and engineering education. 


\section{The use of metacognitive writing-to-learn prompts in an engineering statics class to improve student understanding and performance}

\section{Introduction}

Writing-across-the-curriculum initiatives tend to focus on the idea of using writing as a means to deepen student understanding of concepts and rhetorical practices in the discipline. ${ }^{1,2,3}$ It is common practice in these programs to integrate as much writing as possible in different disciplinary contexts. ${ }^{4,5,6}$ The supposition is that writing naturally enhances learning in most, if not all, learning spaces. This notion dates back to Janet Emig's classic article, "Writing as a Mode of Learning," written in 1977. In brief, Emig argues that writing is profoundly "integrative": it requires competing thought processes to come together as one. This confluence, in itself, enhances learning, according to Emig:

Writing is also integrative in perhaps the most basic possible sense: the organic, the functional. Writing involves the fullest possible functioning of the brain, which entails the active participation in the process of both the left and the right hemispheres. ... Also, a unique form of feedback, as well as reinforcement, exists with writing, because information from the process is immediately and visibly available as that portion of the product already written. The importance for learning of a product in a familiar and available medium for immediate, literal (that is, visual) re-scanning and review cannot perhaps be overstated. ${ }^{5, \text { p. } 125}$

Since writing involves multiple parts of the brain, writing is inherently integrative. The implication of Emig's argument is clear: in whatever form and for whatever purpose, writing necessarily leads to learning.

Accordingly, writing-to-learn strategies have been integrated in science classrooms to enhance understanding of scientific concepts and to encourage metacognition on the part of the students. Hanson and Williams for example, integrated writing-to-learn protocols in their engineering assignments, specifically employing an "explain the problem" technique. ${ }^{2}$ In interviews with students participating in their classes, Hanson and Williams found that writing prompts provided students with a clearer view of their own understanding of engineering problems.

Writing-to-learn exercises are one effective means for encouraging metacognition, as the exercises force students to demonstrate through writing that they either understand the link between the quantitative expression and the concept, or that they need more help in this area., ${ }^{7,}$ 8,9 In the current paper, we describe our experiences integrating a writing-to-learn component into an undergraduate engineering Statics course in an attempt to improve students' metacognition, learning, and class performance. Initially, we asked students to describe their thought processes step-by-step as they worked through homework problems. We have continued to modify the writing prompt each semester after examining the results from each iteration. 


\section{Method}

In our initial iteration, we asked students to respond to a writing prompt (described below) for one specific problem (out of 3-5 total problems) on each of 10 homework assignments per semester. Through student surveys and interviews, we assessed the effect of responding to these prompts on students' perceptions of their understanding of statics concepts and on students' ability to identify when they did not understand a concept. We also assessed whether the inclusion of the writing prompt improved student performance on exams.

Homework was assigned approximately every 1.5 weeks in the lead author's Statics class. The skills required to solve the problem selected on each assignment directly correlated to those needed to solve a problem on the upcoming exam. Performance on the written portion of the homework was worth $20 \%$ of the homework grade to motivate completion of the assignments, and all work was graded by the instructor using an algorithm that was shared with the students. Student surveys were administered twice during the semester to learn more about students' perceptions of the value of the additional writing assignments, and student volunteers were interviewed about their experiences with the writing prompt at the end of the semester.

Control data were collected during two semesters preceding the grant period, when the writingto-learn exercise was not used as part of the course.

\section{Evolution of the writing-to-learn exercise}

The first iteration of the writing prompt had two parts. Part one required students to describe each step of their solution to a homework problem before performing any associated calculations. We hoped that this detailed step-by-step writing protocol would encourage students to consider the purpose of each step of the calculation and thus prevent them from resorting to a "plug-and-chug", approach to solving the problem. Additionally, this structure also enabled separate assessment of a student's ability to conceptualize the problem and perform the mathematical steps of solving the problem. We envisioned that this could provide a means for us to help students more effectively; reviewing their answers for this part would enable us to differentiate between students who could not solve the problem because they did not understand the process for doing so and students who understood how to solve the problem but did not have the math skills to do so.

The second step of the prompt required students to assess whether their numerical answer seemed reasonable. This assessment compelled students to think about the physical meaning of the numbers on the page and to put their answer into broader context. This step, in turn, was designed to facilitate student thinking about the problem in a real-world context, strengthening their understanding of the concepts and providing an approach for recognizing errors. This assessment also encouraged students to examine their own thinking processes. If a student could not rationalize his or her solutions - if he or she could not identify why a solution was right or

\footnotetext{
a By "plug and chug" we mean an approach where students attempt to choose the correct formula for solving a problem and then plug in numbers. This approach usually signals a poor apprehension of the problem and its conceptual underpinnings; students are just trying to make a formula fit, whether or not it is appropriate to answering the question.
} 
wrong-then the student would come face-to-face with a limitation of his or her own understanding of this particular concept or question.

We implemented this intervention for two semesters with a total of 72 students across three sections. Many students indicated on surveys that they found the exercise useful in improving their understanding of statics concepts. However, we had difficulty with compliance with the writing portion of the prompt. Students complained that writing out the steps felt tedious. Further, the writing responses were challenging to grade effectively and consistently, even with a well-defined grading algorithm. It became clear that even if this intervention were effective, it would be unlikely to be broadly implemented due to these challenges. The intervention resulted in only small trends in improved test performance. These observations led us to reconsider and redefine our writing prompt.

After considerable re-assessment, discussion and continued research, we streamlined the writing assignment. In the latest iteration of the prompt, implemented during the Fall 2013 semester, we asked students to reflect on any confusion they had about how to solve the selected homework problem and then revise this reflection after receiving instructor input. Specifically, we had students assess the reasonableness of their numerical answer to each problem and then answer a series of questions to describe any confusion they had about the concepts or computations required to solve the problem. The last of these questions required students to pose questions that, if answered, would help them better understand how to solve the problem. The problem solution was then demonstrated in class as students corrected their own work, during which time students were encouraged to ask the questions they had formulated when they completed the problem at home. Following this demonstration, students were asked to re-examine and revise their conceptual and computational errors in writing while in class. This writing assignment included a place to note any lingering questions. Finally, the instructor provided feedback when grading the homework as to whether students' understanding of the source of any errors was accurate. The instructor also answered any questions students wrote.

Replacing the written description of computation with a written assessment requiring students to describe any confusion they had about the problem offers multiple benefits. First, students' efforts were directed towards an activity that is a more natural part of the problem-solving process and seemed to be more of a clear benefit to them. Second, it provided the instructor with useful information as to what concepts students were struggling with, and it offered an easy mechanism for directly answering individual students' questions when grading their assignments. Finally, if the inclusion of a revision step in this context functioned as reported in other related fields, ${ }^{10,11,12,13}$ then directing students to reflect on the cause of their misunderstanding should lead them to have a better understanding of the concept under review.

We implemented this revised writing intervention in both sections of the lead author's Statics course during the Fall 2013 semester, with a total of 48 students. (Two students' data were excluded from our analyses; one because the student was caught cheating on the final exam, and one because the student had not completed all exams and was taking an Incomplete in the course.) 


\section{Results}

One of our major concerns was the completion rate of the written assignments in the original iteration of the study. Of the 72 students who were required to write out the steps of selected homework problems, only 27 students $(37.5 \%)$ completed all 8 written assignments, with 11 more students (an additional 15.2\%) completing 7 of 8 assignments. In the Fall 2013 version of the study, where students wrote about issues that confused them in the problems, full compliance was improved, but still lower than expected, with only 19 students $(39.6 \%)$ completing every athome assignment, and 9 more completing 9 of 10 of them (an additional 18.8\%). While changing the structure of the writing prompt did not result in as large of an improvement in compliance as we had hoped for (with $58.4 \%$ as opposed to $52.7 \%$ of students not missing more than one assignment), anecdotally, the instructor did receive less negative feedback from students about being asked to complete the assignments. Further, the in-class component of the writing assignment meant that more students did reflect on their understanding of the problems in writing, with $47.9 \%$ of students completing every in-class questionnaire (immediately following review of the problem), and $72.9 \%$ of students missing no more than one questionnaire.

Although the course exams and grading structure were very similar across semesters, we found no differences between overall grades among our pre-intervention group, our first intervention group, and our most recent intervention group, $F(2,150)=0.109, p>0.5$. This lack of effect may be due to the low compliance rates, variability in the utility of the writing intervention for different levels of students, or other factors, such as differences in the background knowledge of the students.

At the same time, students from both intervention groups reported that they found the intervention to be useful, with the majority of students indicating through in-class survey responses that the writing interventions were either "very helpful" or "helpful" in learning the material, in identifying their errors, and in helping them to do well on the exams. In an interview, one student from the second intervention group remarked: "I thought the writing problems made you really think about doing your homework, really made you think about why you had the right answer or not." Another student noted on an in-class survey that he or she "believe[d] the work assigned help[ed] to reinforce the instructions that I have learned in class and has helped motivate the frequent review of material which I may have let slip by not reviewing as often."

From open-ended survey and interview comments, some students indicated that the writing exercises were most helpful when they had some basic idea of how to approach the problem. However, when the students had no conceptual or computational foothold, they found the exercises frustrating. We assume that this is because they didn't have enough knowledge to use the questions to help direct their thinking about the solution to the problem.

One area in which the second intervention does appear to have had a more positive impact than the first is in motivating students to seek assistance when they found themselves having trouble with the homework. In response to survey questions at the end of the semester, $74 \%$ of students (as opposed to $67 \%$ of students from the first intervention group) answered that the assignments made them more likely to consult their textbook or notes, with $74 \%$ (as opposed to $51 \%$ of students from the first intervention group) reporting that the assignments made them more likely 
to seek help from a peer, tutor, or the instructor. This last difference was significant, $\chi 2(1, N=$ $100)=5.71, p=0.017$.

\section{Discussion}

While there is no denying the benefits of writing generally, our experience shows that, at least in the context of our study, writing does not always translate to improved performance on standard engineering exams. In the first iteration of the writing prompt we used, students wrote out the computational steps of an engineering problem and then evaluated the correctness of the problem. We believed that writing out the steps of the problem and evaluating its correctness would concretize both procedural and conceptual knowledge and lead students to greater metacognitive apprehension of the concepts under consideration, as well as their own understanding of these concepts (and attendant mathematical calculations). This activity, however, did not lead to discernible metacognitive results and was frustrating to complete for the students. From this experience, we hypothesized that writing interventions would be more successful if they were more specific and targeted to both the practices and experiences of students. This realization challenges past practices of integrating writing-to-learn exercises in non-literary domains. $5,6,14$

Recent studies on the use of writing-to-learn assignments in math, science and engineering courses have supported our observations; researchers have found that it is exceedingly difficult to identify the kinds of writing that lead to increased student performance and metacognitive understanding. ${ }^{10,11}$ Nevertheless, writing across the curriculum initiatives tend to focus on the idea of using writing as a means to deepen student understanding of concepts and rhetorical practices in the discipline. ${ }^{1,6,2,15}$ Even though results in these studies have been inconsistent at best regarding the efficacy of writing, the assumption persists that writing is beneficial in most, if not all, cases.

Recent research in cognitive psychology may provide potentially useful heuristics for the integration of writing into an engineering context, indicating that written learning prompts or exercises fitted to a students' specific learning context may lead them to more substantive and measurable mastery of material. Nokes et al., "for example, note the importance of "fit" in designing heuristic models, writing,

[W]e posit the instructional fit hypothesis, which is the idea that the cognitive processes triggered by an instructional intervention must match, or fit, the learning scenario (i.e., learner's prior knowledge, the task structure, and the knowledge required to perform well on the task). In the case of self-explanation, we hypothesize that the gap-filling and mental-model revision prompts are best suited for particular types of learning scenarios that depend on the student's prior knowledge (e.g., whether or not they have a prior misconception), the relation between that knowledge and the task domain, and the structure of the task or learning activity. ${ }^{16, \text { p. } 649}$

Writing interventions, then, must match not just the new knowledge context, but also the old: students' past learning experience must be factored into designing heuristic interventions if they are to work effectively, as the knowledge students bring to a problem affects their approach. This effect is demonstrated most clearly in studies comparing experts and novices. For example, 
physics experts classify problems by their underlying physics principles, such as whether the problems involve applying conservation of linear momentum or equations of motion, while novices classify them based on surface similarities, such as whether problems involve springs or inclined planes. ${ }^{17}$ Thus, different pedagogical approaches are likely to be helpful to students at different stages of understanding, and the writing-to-learn component may only be effective for a subset of students with a basic level of understanding. Students with too little background knowledge may not know enough to find extra articulation of their confusion helpful if they do not know where to begin. Students with strong background knowledge may not find the writing helpful if they already know how to solve the problem and thus do not benefit from additional reflection.

Nokes-Malach et al. ${ }^{18}$ expand upon this notion of "fit." In brief, their article describes the efficacy of self-explanation prompts and other heuristic tools in encouraging learning that may transfer easily to other problem sets within the discipline itself and to encourage an understanding of the relationship between "domain principles" and "problem features." Various heuristic tools are examined, including self-explanation prompts, analogical comparison of worked examples, and reading "worked examples" as models to solve "practice problems." The authors conclude that self-explanation prompts, in particular, are most effective in encouraging an understanding of domain principles, accessing prior and current knowledge, and facilitating near and far transfer. Self-explanation prompts require that the student "explain to oneself with the goal of making sense of new information."18 These prompts also implicitly compel students to evaluate the answers to their own problems, thus requiring students to engage in a metacognitive analysis of their work. Nokes-Malach et al. ${ }^{18}$ found that good learners who use self-explanation prompts generate more inferences, and were able to explain when the solution was applicable to other problems and when it was not. Poor learners were unable to use the prompts in this way. Thus, good learners could use self-explanations to revise their understanding and reduce their misconceptions about a topic.

In writing studies, revision - or the opportunity to reexamine and reevaluate an argument in writing - has similar features to self-explanation prompts. The "second look" that revision requires allows students to reframe the questions generated by the writing prompt itself and to generate new information from reevaluating their written work. A revision step allows students to more fully access the mental steps that cognitive science researchers view as critically important to learning effectively.

These ideas are what motivated our redesign of the writing prompt to require students to describe their misconceptions and revisit them after instructor input. While this approach was more positively received than our earlier interventions, it did not noticeably affect course performance, which may continue to be related to poor compliance. We intend to address this in future semesters by having all students' writing occur in class while homework is being graded. Specifically, students will be asked to write down what open questions they have about the problem they completed at home. They will then observe the solution to the problem and have the opportunity to ask their questions. Finally, they will write how their understanding of the problem has changed after seeing the solution. We anticipate that consolidating all of the writing into a single in-class setting will improve the quantity and quality of student responses and will have an increased impact on student metacognition and performance. 
Other possible modifications involve the inclusion of some peer-work during the writing intervention. We may have students work in pairs and exchange their questions with each other. Students will be encouraged to try to answer their peers' questions in writing before the solution is provided to them by the instructor. This collaborative peer-work should allow students not only to focus on their understanding of a particular problem, but to also have an "audience" for their work in mind. Having an audience in mind while composing has been shown to increase the analytical sophistication of student writing in general. ${ }^{18,19,20}$ Students simply do not resort to a convenient short-hand while writing, but try to give their reader a fuller experience of their thinking process. We hope that having an "in-built" audience of peers will formalize this exercise for the student writers and lead to greater analytical and communicative accountability.

In sum, we are in the middle of a learning process ourselves as we continue refinements of an intervention designed to improve student metacognition, and in turn, student learning. Writing can be an important method for helping students to reflect on their understanding of a problem. However, it is clear that the simple addition of a writing-to-learn component is not sufficient for affecting student performance in this context. Further, our goal is to help find practical methods that can improve student understanding through more efficient uses of study and class time, without requiring excessive extra work on the part of either the students or the instructor. It is a challenge, but we look forward to continued discussion and exploration on this important issue.

\section{Acknowledgement}

This work was supported by the National Science Foundation's Research Initiation Grants in Engineering Education under award No. 1137009. Any opinions, findings, and conclusions or recommendations expressed in this material are those of the authors, and do not necessarily reflect the views of the National Science Foundation. The authors thank Dr. M. David Burghardt and Dr. Sheri Sheppard for their input on this project. They also thank Angela Miller for assistance with data entry.

1. Case, J. et al. 2003. Approaches to learning in a second year chemical engineering course. International Journal of Science Education 25(7): 801-819.

2. Hanson, J. H. et al. 2008. Using writing assessments to improve students' self assessment and communication in an Engineering statics course. Journal of Engineering Education. 97(4): 515-529.

3. Beall, H. 1998. Expanding the scope of writing in chemical education. Journal of Science Education and Technology, 7(3): 259-270.

4. Bazerman, C and Russell, D.R. (eds.) 1994. Landmark essays on writing across the curriculum. Hermagoras Press, Davis, CA.

5. Emig, J. 1977. Writing as a mode of learning. College Composition and Communication 28(2): $122-128$.

6. Fulwiler, T. \& Young, A. (eds.) 2000. Language connections: Writing and reading across the curriculum. WAC Clearinghouse Landmark Publications in Writing Studies, Fort Collins, CO. 
7. Berkenkotter, C. 2000. Writing and problem solving. In Toby Fulwiler and Art Young (eds.), Language connections: Writing and reading across the curriculum. p. 33-44. WAC Clearinghouse Landmark Publications in Writing Studies, Fort Collins, CO.

8. Poe, M. 2000. On writing Instruction and a short game of chess: Connecting multiple ways of knowing and the writing process. Language and Learning Across the Disciplines 4(1): 30-44.

9. Rich, J., et al. 2010. From concept to application: Student narratives of problem-solving as a basis for writing assignments in science classes. Across the Disciplines 8(1) Retrieved from $\mathrm{http}: / /$ wac.colostate.edu/atd/articles/richetal2011.cfm.

10. Kagesten, O., et al. 2006. Supplementary explanations in undergraduate mathematics assessment: A forced formative writing activity. European Journal of Engineering Education, 31(6): 705-715.

11. Porter, M. et al. 2000. Examining the effects of writing on conceptual and procedural knowledge in calculus. Educational Studies in Mathematics. 42: 165-177.

12. Pugalee, D. 2004. A comparison of verbal and written descriptions of students' problem-solving processes. Educational Studies in Mathematics, 55(1/3): 27-47.

13. Miller, D. L. 1992. Teacher benefits from using impromptu writing prompts in algebra classes. Journal for Research in Mathematics 23(4): 329-340.

14. Merrill, Y. 2004. Writing as situated thinking in general education. Across the Disciplines, 1 . Retrieved from http://wac.colostate.edu/atd/articles/merrill.cfm.

15. Kokkala, I. et al. 2003. Writing science effectively: Biology and English students in an author-editor relationship. Journal of College Science Teaching, 32(4): 252-257.

16. Nokes, T.J., et al. 2011. Testing the instructional fit hypothesis: The case for self-explanation prompts. Instructional Science 39: 645-666.

17. Chi, M. T. H., et al. 1981. Categorization and representation of physics problems by experts and novices. Cognitive Science, 5: 121-152.

18. Cho, Y. H et al. 2011. Peer reviewers learn from giving comments. Instructional Science 39: 629-643.

19. Wheeler, E. et al. October 2000. Writing in engineering courses. Journal of Engineering Education: 481-486.

20. Klein, P.D. 1999. Reopening inquiry into cognitive processes in writing-to-learn. Educational Psychology Review 11(3): 203-270. 